Cahiers $d u$ MONDE RUSSE

\section{Cahiers du monde russe}

Russie - Empire russe - Union soviétique et États indépendants

43/2-3 | 2002

Contacts intellectuels, réseaux, relations internationales

\title{
Les archives du comité directeur du ZEMGOR
}

\section{Catherine Gousseff et OLGA PICHON-BOBRINSKOY}

\section{(2) OpenEdition}

\section{Journals}

Édition électronique

URL : https://journals.openedition.org/monderusse/8515

DOl : 10.4000/monderusse.8515

ISSN : $1777-5388$

Éditeur

Éditions de l'EHESS

Édition imprimée

Date de publication : 1 avril 2002

Pagination : 529-544

ISBN : 2-7132-1781-4

ISSN : $1252-6576$

Référence électronique

Catherine Gousseff et OLGA PICHON-BOBRINSKOY, « Les archives du comité directeur du ZEMGOR », Cahiers du monde russe [En ligne], 43/2-3 | 2002, mis en ligne le 01 janvier 2007, consulté le 03 septembre 2022. URL : http://journals.openedition.org/monderusse/8515; DOl : https://doi.org/ $10.4000 /$ monderusse.8515 
chercher : repérer : avancer

Cet article est disponible en ligne à l'adresse :

http://www.cairn.info/article.php?ID REVUE=CMR\&ID NUMPUBLIE=CMR 432\&ID ARTICLE=CMR 4320529

Les archives du comité directeur du ZEMGOR

par Catherine GOUSSEFF et Olga PICHON-BOBRINSKOY

\section{Editions de I'EHESS | Cahiers du monde russe}

2002/2-3 - Vol 43

ISSN 1252-6576 | ISBN 2713217814 | pages 529 à 544

Pour citer cet article :

-GOUSSEFF C. et PICHON-BOBRINSKOY O., Les archives du comité directeur du ZEMGOR, Cahiers du monde russe 2002/ 2-3, Vol 43, p. 529-544.

Distribution électronique Cairn pour les Editions de l'EHESS.

(C) Editions de l'EHESS. Tous droits réservés pour tous pays.

La reproduction ou représentation de cet article, notamment par photocopie, n'est autorisée que dans les limites des conditions générales d'utilisation du site ou, le cas échéant, des conditions générales de la licence souscrite par votre établissement. Toute autre reproduction ou représentation, en tout ou partie, sous quelque forme et de quelque manière que ce soit, est interdite sauf accord préalable et écrit de l'éditeur, en dehors des cas prévus par la législation en vigueur en France. Il est précisé que son stockage dans une base de données est également interdit. 


\section{LES ARCHIVES DU COMITÉ DIRECTEUR DU ZEMGOR}

Les archives du Comité directeur du Zemgor (localisé à Paris) ont été déposées au centre des archives russes de l'université de Leeds en 1987, à la demande du président du Zemgor. Elles n'ont pas encore fait l'objet d'un inventaire. Le but de cette présentation est donc de préciser le contenu de ce fonds ainsi que d'esquisser les différentes directions de recherche qui peuvent être envisagées à partir des documents existants.

\section{Présentation historique du Zemgor}

Le «Zemsko-gorodskoj komitet», plus connu sous le nom de Zemgor (traduit en français par «Comité des zemstvos et villes russes de secours aux citoyens russes à l'étranger »), s'est constitué en association (loi 1901) en février 1921, se définissant comme une organisation à caractère exclusivement humanitaire d'aide aux réfugiés issus de la Révolution et de la guerre civile. Cette association se présentait néanmoins comme le prolongement des anciennes administrations locales russes (zemstva et municipalités) regroupées en Unions en 1914. L'Union des villes et l'Union des zemstva étaient devenues, durant la Grande Guerre, deux importantes organisations para-gouvernementales essentiellement conçues dans le but de pallier les carences de l'État dans le domaine sanitaire et l'assistance aux populations civiles victimes du conflit. Elles furent ainsi amenées à constituer d'importants réseaux hospitaliers destinés aux soldats évacués des fronts, à prendre en charge, au moins partiellement, les réfugiés en provenance des régions occidentales occupées par les armées des puissances centrales (création de points d'accueil de transit, enregistrement et aide au placement des réfugiés dans les différentes régions de Russie).

Tout en conservant chacune leur indépendance, les deux Unions ont été amenées à collaborer étroitement dans deux domaines principaux : l'assistance aux 
réfugiés qui entraîna la création d'un comité conjoint (le Sobež) ; le ravitaillement de l'armée en petit matériel de guerre (munitions, masques à gaz, notamment), en fournitures médico-sanitaires et l'aménagement des fronts (tranchées, en particulier) ; cette collaboration fut à l'origine de la création du Zemgor proprement dit, ce comité regroupant des membres de l'une et l'autre des Unions.

Officiellement liquidées en 1919 par le gouvernement bolchevik, l'Union des zemstva et l'Union des villes avaient été entravées dans leurs activités dès 1918. Au cours de la guerre civile, les différents animateurs de ces organisations reconstituèrent dans les régions contrôlées par les forces anti-bolcheviques (Sibérie, régions méridionales, Crimée) des comités provisoires qui poursuivirent l'action sanitaire en faveur des armées et des populations civiles. En 1920, lors des différentes évacuations de l'Armée blanche des rives de la mer Noire, de très nombreux membres, à l'échelle pan-russe (Union), ou locale (zemstvo, ville), se retrouvèrent en exil.

Par ailleurs, l'Union des zemstva et l'Union des villes avaient créé au cours de la Première Guerre mondiale des représentations dans différents pays alliés afin d'obtenir des soutiens et de coordonner l'effort de guerre. Des réseaux se constituèrent avec des organisations caritatives occidentales, voire avec différents interlocuteurs gouvernementaux.

Ainsi de nombreux aspects de l'activité des Unions prédisposaient ces organisations à jouer un rôle central dans l'histoire de l'émigration russe : l'expérience acquise dans le domaine humanitaire au cours de la guerre et de la guerre civile (notamment à l'égard des réfugiés internes de l'empire russe) ; de nombreuses relations nouées en Europe occidentale avec des acteurs associatifs et étatiques ; enfin la proximité politique des responsables des Unions (pour la plupart libéraux, issus du parti K-D, octobriste ou du parti S-R) avec les représentants officiels du Gouvernement Provisoire à l'étranger.

Ces derniers, ambassadeurs et diplomates, jouissaient d'une certaine autorité auprès des puissances alliées en l'absence de reconnaissance du gouvernement bolchevik. Au début des années 1920, ils incarnaient, de facto, les seules autorités mandatées du dernier gouvernement russe et pouvaient, à ce titre, prétendre disposer des fonds de l'État russe à l'étranger. Constitués en «Conseil des ambassadeurs », ces représentants définirent les principes directeurs de l'organisation de l'aide aux réfugiés russes dispersés dans les différents États européens en assignant à deux organisations principales les tâches d'assistance : la Croix-Rouge russe fut désignée pour prendre en charge l'assistance sanitaire des réfugiés; le Zemgor fut appelé à s'occuper de l'enregistrement des personnes, de l'aide au placement dans différents pays, de l'aide à l'insertion et à la formation professionnelle. D'autre part, il joua un rôle cardinal dans la création d'institutions scolaires et d'établissements d'enseignement supérieur.

Il semble ainsi que la création du Zemgor, en tant qu'institution, ait résulté de la nécessité de centraliser et de coordonner l'activité des différents comités, de zemstva d'une part, et/ou de villes de l'autre, reconstitués dans plusieurs pays, au gré des opportunités, notamment grâce à la présence locale des anciens membres de l'une au l'autre Union. En effet, dès sa création, le Zemgor s'est présenté comme un 
organe fédérateur des différents comités existants et, en définitive, comme le Comité directeur des actions engagées par ces derniers. Les statuts de fondation du Zemgor précisent que toute association affiliée à l'organisation ne peut agir que sous mandat du Zemgor et s'engage à rendre régulièrement compte de ses activités. Organisation conçue dans un contexte diasporique, le Zemgor a progressivement perdu ses liens avec les différents comités dispersés en Europe dans la deuxième moitié des années 1930 mais il a en revanche poursuivi ses activités en France jusqu'à nos jours.

\section{Présentation générale des archives}

Les archives du Zemgor rendent compte de la très grande diversité des préoccupations de l'organisation. Elles permettent d'aborder à la fois l'histoire sociale, organisationnelle de l'émigration mais aussi certains événements de l'histoire soviétique dans lesquels le Zemgor a été, directement ou non, impliqué. L'insurrection de Kronstadt, la famine de 1921-1922, la collectivisation de la fin des années 1920 et les migrations spontanées vers la Pologne qui en ont résulté constituent ainsi quelques-uns des thèmes touchant à l'histoire de l'URSS.

S'agissant de l'histoire de l'émigration russe, les archives du Zemgor constituent une source très précieuse et particulièrement variée. Elles concernent notamment la situation des réfugiés dans les différents pays d'accueil européens, les actions d'aide mises en place, les relations entretenues avec les différents gouvernements des États d'accueil (notamment par le biais du placement des émigrés), les collaborations mises en œuvre avec la Société des nations (Haut commissariat aux réfugiés - HCR) et le Bureau international du travail (BIT), les relations entre les différents représentants de l'émigration (Conseil des ambassadeurs, Office central des réfugiés russes, Croix-Rouge russe, etc.).

Les matériaux conservés dans ce fonds sont d'ordre très divers : rapports, correspondances, comptes rendus de réunions, fichiers et listes de réfugiés enregistrés à diverses fins, enquêtes menées auprès de différentes institutions, bilans financiers, revues de presse et bulletins associatifs, photographies, statistiques et graphiques (démographie, état sanitaire des réfugiés), cartes (par exemple, carte de l'organisation du corps du Don sur l'île de Lemnos), documents juridiques (conventions sur les réfugiés, notamment), etc.

Ce fonds reflète assez fidèlement l'évolution de l'histoire communautaire de l'émigration. Particulièrement riche en ce qui concerne le début des années 1920 , il contient également de très nombreux documents pour la période qui s'étend jusqu'au milieu des années 1930. En revanche, à partir de 1935 environ, les documents deviennent plus lacunaires. Pour la période de la Seconde Guerre mondiale, la documentation n'est constituée, semble-t-il, que de quelques dossiers de correspondances. Les archives concernant l'après-guerre, qui représentent environ $20 \%$ de l'ensemble, reflètent, pour l'essentiel, l'activité du Zemgor de France. 
Les archives du Zemgor n'ont pas encore fait l'objet d'un inventaire et sont rangées par cartons sur huit étagères d'entrepôt facilement accessibles. Néanmoins, une partie des archives de l'association avait déjà fait l'objet d'un classement (documents répertoriés chronologiquement dans des classeurs thématiques). Il s'agit, pour l'essentiel, de documents portant sur les pays où le Zemgor était représenté (rapports entre le Zemgor de Paris et l'organisme affilié), ou portant sur des activités spécifiques du Zemgor (placement des réfugiés dans les régions agricoles du Sud-Ouest, par exemple). Il existe en outre un certain nombre de cartons pré-classés de façon thématique (Ligue des nations, finances, photographies, produits du timbre Nansen, Croix-Rouge, etc.) et plusieurs cartons de correspondances identifiées (par exemple Bahmetev, L'vov, Rubinštejn). Il faut signaler l'existence de certaines archives personnelles, telles celle de Rudnev, l'un des grands responsables du Zemgor, ou encore diverses correspondances de Giers (membre du Conseil des ambassadeurs dont la plupart des archives personnelles se trouvent à l'Insitut Hoover). Parmi les archives présentes, mais ne relevant pas, à proprement parler, des archives du Zemgor se trouvent également des correspondances télégraphiques datant de la guerre civile ainsi que divers documents de la même époque. Quelques cartons seulement contiennent des documents très hétérogènes (par la provenance et l'époque). Ainsi, en dépit de l'absence d'un inventaire, le fonds s'avère, en l'état, rapidement identifiable dans ses différentes composantes.

\section{Axes de recherches envisagés à partir des archives du Zemgor}

Plutôt que de procéder à une description thématique du fonds du Zemgor de Leeds, il a paru plus pertinent d'esquisser les principales pistes de recherches qui se dégagent du contenu documentaire existant, qui complète dans bien des cas d'autres sources telles que la collection des Archives de Prague conservées au Archives d'État de la Fédération de Russie (GARF) de Moscou et les Archives de la Société des nations (SDN) conservées à Genève, les archives de l'émigration conservées dans différents centres aux Etats-Unis (Hoover, Archives Bakhmetiev de l'université de Columbia notamment).

\section{I - Le Zemgor, l'histoire d'une administration en diaspora}

En dépit du changement radical de contexte, de la Russie à l'émigration, le Zemgor a continué de fonctionner comme une véritable administration au service des réfugiés, développant un capital de pratiques et d'expériences acquises au cours de la guerre dans le domaine humanitaire. En même temps, la réalité de l'exil, la mutation du Zemgor en pur organisme d'assistance, dépendant dans la portée de ses actions d'interlocuteurs multiples et nouveaux, ont radicalement transformé les fondements de cette organisation. L'étude du Zemgor comme administration, à 
travers sa structure, ses orientations, les parcours des hommes qui l'ont animé, devrait contribuer à préciser, par delà la rupture de l'exil, la part de continuité, d'héritages de la Russie à l'émigration, qui constitue la grande singularité de l'histoire du Zemgor.

\section{Le fonctionnement du Comité directeur}

L'ensemble documentaire permet de reconstituer le mode de fonctionnement du Zemgor qui s'apparente à une véritable institution, très hiérarchisée, depuis son Comité directeur parisien (et les différents départements qui le composaient), jusqu'aux comités dispersés dans les différents États d'accueil qui lui étaient affiliés.

La rétrospective du fonctionnement, de l'action et de l'évolution du Comité directeur du Zemgor peut être entreprise en premier lieu à l'aide des comptes rendus des réunions, sortes de bulletins internes de périodicité très fréquente (une fois par semaine au moins), qui détaillaient également l'activité des différentes commissions attachées au Comité directeur. Ces commissions ont évolué selon les périodes. En 1922, il existait huit commissions ou départements : financier, de ravitaillement, de colonisation, du travail, de l'enseignement, département médicosanitaire, d'informations, de l'administration générale.

Outre les bulletins susmentionnés, de nombreux documents de nature diverse (publications du Zemgor, correspondances entre membres du Zemgor, mémorandums) permettent d'aborder sous d'autres angles (représentation vis-à-vis des interlocuteurs extérieurs notamment ou, au contraire, relations internes, conflits) l'existence du Comité directeur parisien.

Dans tous les champs d'action définis, qu'il s'agisse de l'éducation, de l'aide à l'enfance, à l'insertion professionnelle, le Zemgor a repris les pratiques développées par les Unions des villes et des zemstva durant la guerre en commençant par identifier précisément les populations concernées, leurs conditions et leurs besoins. D'où la priorité accordée au travail d'enquêtes, de collectes d'informations statistiques, de rapports spécifiques dans l'objectif de rationaliser les formes d'assistance. De ce fait, les archives de Leeds contiennent de très nombreux ensembles documentaires thématiques - questionnaires, rapports préliminaires et définitifs - qui permettent de retracer l'élaboration des sources d'informations. À titre illustratif, on peut citer l'enquête effectuée par le Zemgor à la fin des années 1920 concernant la situation des enfants de réfugiés en Europe. Les archives de Leeds contiennent en effet l'ensemble des questionnaires diffusés auprès d'interlocuteurs communautaires, et le rapport d'enquête présenté au Sous-comité consultatif pour l'étude de la condition des enfants réfugiés auprès du Haut commissariat aux réfugiés. De manière générale, les archives portent l'empreinte de l'importance du rapport comme instrument de planification des actions, comme instrument de visibilité, de transparence vis-à-vis des institutions extérieures de soutien et vis-à-vis de la communauté russe en exil. 


\section{La dimension diasporique du Zemgor}

L'action du Comité directeur peut être, par ailleurs, précisée à travers son travail de coordination avec les comités locaux. Dans les années 1920, le Zemgor était représenté dans 17 pays ou régions : France, Grande-Bretagne, Allemagne, Finlande, Estonie, Lettonie, Lituanie, Tchécoslovaquie, Pologne, Suisse, Yougoslavie, Bulgarie, Turquie, Egypte, île de Chypre, Grèce, Tunisie. Les comités affiliés au Zemgor étaient soit des comités de l'Union des villes, soit des comités de l'Union des zemstva, soit des comités de l'Union des villes et des zemstva, soit des comités constitués par des représentants directs du Zemgor de France, soit encore des comités regroupant différentes organisations philantropiques russes constituées localement. Il semble que la définition des comités locaux (Union des villes ou Union des zemstva en particulier) ait été fonction de la représentation de leurs membres. Dans les cas, par exemple, où les animateurs locaux provenaient essentiellement de l'administration des anciens zemtsva, le comité constitué était alors un comité de l'Union des zemstva.

Les archives regroupées dans les classeurs rendent compte des correspondances régulières entre le Comité directeur et ses filiales, du mode de contrôle excercé par le Zemgor de Paris, des orientations proposées, des requêtes provenant de part et d'autre, des difficultés rencontrées par les comités locaux. Le rôle d'arbitrage du Comité directeur est particulièrement sensible dans les questions d'ordre financier, et dans les conflits internes (comme en Allemagne, entre le comité de Berlin et les autres comités implantés sur le territoire de la République de Weimar). Il ressort également de ces correspondances que le Zemgor de Paris (jusqu'au milieu des années 1920 au moins) ne cesse de demander de nouvelles informations sur la situation des réfugiés dans les différents pays, ce qui conduisit certains comités à rédiger des rapports réguliers, sortes de chroniques de l'évolution des différentes colonies émigrées locales, de leurs profils sociaux, de leur mode d'insertion, de leurs besoins, etc.

L'un des sujets les plus fréquemment abordés dans les correspondances entre la direction du Zemgor et les comités locaux concerne, à l'évidence, les questions financières. Toute demande adressée à Paris est présentée sous forme de budget prévisionnel argumenté qui, une fois accepté et dépensé, donne lieu à un rapport financier. Il est ainsi possible de reconstituer le volume des dépenses engagées par le Comité directeur selon les types d'actions et les pays (outre les rapports généraux publiés certaines années par le Comité directeur).

Les correspondances entre Paris et les comités locaux rendent compte indirectement des profils de ces derniers, de leur longévité, de l'étendue de leurs actions. Certains, tel le Zemgor de Prague, sont connus pour leur rôle cardinal dans l'organisation de l'émigration russe à l'échelle nationale (tchécoslovaque en l'occurrence), d'autres en revanche semblent n'avoir connu qu'une existence éphémère (comme en Grèce ou en Finlande). Dans tous les cas, le passage des années 20 à 30 apparaît comme un tournant qui amorce le déclin des filiales du Zemgor en Europe. 


\section{Le financement du Zemgor}

Un ensemble documentaire très varié porte sur les finances du Zemgor (outre les rapports financiers des comités locaux susmentionnés). Correspondances administratives avec différentes banques, correspondances avec le HCR, le BIT, le Conseil des ambassadeurs devraient permettre de reconstituer l'origine des fonds, importants, de cette organisation et par conséquent d'identifier plus finement les grands réseaux de soutien du Zemgor, la part résultant des fonds de l'ancien gouvernement russe placés à l'étranger, celle relevant d'aides gouvernementales spécifiques, de grandes organisations internationales (SDN, BIT), de l'autogestion des réfugiés (timbres Nansen, en particulier), ainsi que de différentes associations caritatives, philantropiques, européennes et américaines, etc.

\section{Les animateurs du Zemgor}

Les archives de Leeds permettent difficilement de retracer les profils et, plus généralement, d'approcher le personnel du Zemgor, en l'absence de tout fichier, de tout dossier spécifique à ce sujet. Combien de personnes ont été salariées par le Zemgor, quels ont été leurs parcours professionnels, leurs origines sociales, quelle a été la part du personnel bénévole ? Ces questions restent ouvertes. Néanmoins, une évaluation reste envisageable, à travers par exemple les bilans annuels d'activités (du Zemgor et des comités locaux) qui dressent un rapport particulier sur les frais de fonctionnement (salaires du personnel, location immobilière, etc).

En ce qui concerne les animateurs du Comité directeur ${ }^{1}$ et des comités locaux du Zemgor, l'analyse des parcours est généralement facilitée par la notoriété de ces responsables, à commencer par son président, le prince G. E. L'vov, qui exerça cette fonction jusqu'à sa disparition, en 1925. Membre du parti K-D, ancien président du directoire du zemstvo de la province de Toula (1900-1903), le prince L'vov devint député de la première douma en 1906. Délégué de l’Union des zemstva pendant la Première Guerre mondiale, il fut chargé de l'assistance aux malades et blessés de guerre puis, lors du renversement de l'autocratie, il s'imposa comme l'une des personnalités politiques de premier plan, devenant président du Gouvernement Provisoire de mars à juillet 1917. A. Konovalov, qui succéda à L'vov à partir de 1925, avait été, quant à lui, un grand industriel du textile de la région de Moscou. Membre fondateur du parti libéral des industriels, il devint au cours de la Grande Guerre vice-président du Comité central des industries de guerre, fonction

1. En 1922, le comité directeur était composé de 30 membres qui étaient (outre ceux cités dans le texte): G. A. Alekseev, N. N. Bogdanov, M. V. Brajkevič, P. A. Buryškin, M. M. Vinaver, V. V. Vyrubov, N. V. Dmitriev, L. L. Katuar, I. N. Kovarskij, K. R. Krovopuskov, N. V. Makeev, I. S. Minor, V. A. Obolenskij, A.S. Orlov, T. I. Pol'ner, E. F. Rogovskij, Ja. L. Rubinštejn, A. A. Titov, S.P. Tjurin, V.A. Harlamov, A.S. Hripunov, N. V. Čajkovskij, F V. Šlippe, P. P. Jurenev, I. A. Kirilov. 
qui le conduisit au poste de ministre du Commerce et de l'Industrie du Gouvernement Provisoire dès mars 1917, peu avant son entrée au comité central du parti K-D. À Paris où il émigra en 1920, Konovalov devint l'un des grands acteurs de la vie associative et culturelle de l'émigration russe. Membre de plusieurs organisations d'entraide en dehors du Zemgor où il était l'une des personnalités les plus impliquées, il fit également partie de la direction du quotidien Poslednie novosti de 1924 à 1940, du conseil pédagogique de l'Institut commercial russe de Paris, de la Société musicale russe à l'étranger, etc. Ses différentes activités reflétaient bien un engagement multiforme dans la vie communautaire de l'émigration, caractéristique qu'il partagea avec nombre de responsables du Zemgor, tel, par exemple, V.F. Zeeler. Ce juriste, membre du parti K-D, ancien maire de Rostov-sur-le-Don (1917) et bref ministre de l'Intérieur dans le gouvernement de Denikin (1920), fut non seulement l'une des personnalités les plus actives du comité directeur du Zemgor, mais également l'un des membres fondateurs du bureau de l'Union des écrivains et journalistes russes à l'étranger, le secrétaire de l'Union des avocats russes en France, l'un des experts russes de la commission consultative pour l'élaboration du statut de réfugié, etc.

Contrairement à la plupart des personnalités du Comité directeur, V. V. Rudnev et $\mathrm{N}$. Avksent'ev venaient, eux, du parti S-R. Rudnev y avait même été membre du comité central. Maire de Moscou en 1917 et député de l'éphémère Assemblée constituante, il émigra à Paris en 1919, se consacra à l'action humanitaire, mais participa aussi à plusieurs entreprises éditoriales et intellectuelles de l'émigration. Secrétaire de la revue Evrejskaja tribuna de 1920 à 1924, il fut également, avec Avksent'ev, l'un des fondateurs des Sovremennye zapiski, la plus grande des revues politico-littéraires de l'émigration.

Parmi les représentants des comités nationaux, les plus notoires furent, à l'évidence, les animateurs du célèbre Zemgor de Prague qui fut à l'origine des initiatives les plus brillantes et durables de la vie culturelle de l'émigration. Son fondateur et président V.M. Zenzinov, qui avait été membre du directoire du gouvernement d'Oufa, était resté un S-R convaincu. Ses liens très étroits avec l'équipe gouvernementale de Prague dataient de la guerre civile où il s'était trouvé en contact avec les responsables de la légion tchécoslovaque. En exil, Zenzinov déploya de multiples activités aussi bien au sein du Zemgor que de la vie politique et intellectuelle de l'émigration où il fut, notamment, un publiciste de grande envergure, collaborant à de très nombreux journaux et revues (Dni, Novaja Rossija, Volja Rossii, etc.).

N. N. Astrov, qui avait été au cours de la Grande Guerre membre du Comité directeur de l'Union des villes, fut à Prague l'un des initiateurs de la création du Centre des archives russes à l'étranger (alias les Archives de Prague, par la suite transférées, pour l'essentiel, en URSS) qui demeure jusqu'à aujourd'hui la plus grande collection d'archives de l'émigration russe. Bien d'autres personnalités mériteraient d'être citées parmi les animateurs du Zemgor. Mais le rappel de ces quelques parcours témoigne assez de la stature des responsables de cette organisation, généralement mieux connus pour leur activité politique et leur rayonne- 
ment intellectuel que pour leur engagement social et communautaire qui reste pourtant un trait spécifique de leur itinéraire en exil. De ce point de vue, les archives conservées à Leeds sont d'un apport essentiel pour préciser le rôle joué par ces élites dans les différentes formes d'actions engagées par le Zemgor. S'il s'agit, en définitive, d'élaborer une sorte de biographie collective des hommes du Zemgor, la collection de Leeds vient en complément des sources existantes et requises pour la recherche, qu'il s'agisse des dossiers personnels et du fonds autobiographique des archives du GARF, des archives de l'émigration conservées à l'Institut Hoover de l'université de Stanford ou d'autres centres encore. Les fonds des Unions des zemstva et des villes, conservés aux Archives historiques militaires de Russie (Rossijskij gosudarstvennyj voenno-istorčeskij arhiv - RGVIA) permettent, quant à eux, de retracer les parcours de nombreux responsables du Zemgor pendant la guerre.

\section{II - Le Zemgor, un éclairage sur l'histoire sociale et culturelle de l'émigration russe}

L'étude de l'action du Zemgor comporte de nombreux enseignements sur des aspects mal connus de l'histoire sociale, communautaire, de l'émigration et permet de préciser sa contribution au développement culturel des Russes en exil.

Dans l'esprit des animateurs du Zemgor, l'aide purement humanitaire et l'action culturelle étaient conçues dans une relation très étroite et de forte complémentarité, car ils conféraient à la culture un rôle réparateur, la concevaient comme un soutien psychologique et moral essentiel dans l'état de déracinement et de désarroi où se trouvaient les réfugiés.

La particularité du positionnement du Zemgor par rapport aux nombreuses organisations russes émigrées est d'avoir conçu son action dans une double perspective, de maintien identitaire et de renforcement du lien communautaire d'une part, et, d'autre part, d'adaptation aux différents pays d'accueil. Ce dernier aspect distingue en effet le Zemgor au sein de la vie associative de l'émigration qui est restée, elle, essentiellement orientée vers la conservation et le développement du patrimoine culturel russe. L'action sociale du Zemgor en faveur de l'insertion des réfugiés (emplois, placements collectifs et individuels, formations professionnelles, etc.) offre, du point de vue de l'histoire sociale de l'émigration russe, différents éclairages autant sur la vie interne de la communauté, les formes d'autogestion de l'aide aux réfugiés, que sur les profils sociaux des réfugiés eux-mêmes et leurs besoins ou encore sur les liens, les réseaux constitués entre le Zemgor et les interlocuteurs extérieurs (pouvoirs publics, organismes internationaux). Enfin, cette action permet d'identifier les grands acteurs sociaux de l'émigration, reconnus pour leur appartenance à l'élite intellectuelle exilée, mais mal connus jusqu'à présent pour leur engagement et leur rôle central dans l'organisation sociale et communautaire des Russes en exil. 


\section{Le rôle du Zemgor dans l'organisation communautaire de l'émigration russe}

La vie associative de l'émigration russe, bien que souvent mentionnée pour la diversité et la multiplicité de ses cercles, unions, sociétés, associations, n'a jamais fait l'objet d'une étude approfondie. Or, le Zemgor offre un point de vue pertinent sur cet aspect caractéristique de l'émigration russe. Sa position, ses mandats permettent d'apprécier l'existence d'une hiérarchie interne forte, depuis le Conseil des ambassadeurs qui a été une sorte de substitut au Gouvernement Provisoire face aux interlocuteurs étrangers (et, dans les premières années de l'émigration, le commandement Wrangel avec lequel le Zemgor s'est trouvé en relations étroites bien que tendues), jusqu'aux multiples associations d'entraide que le Zemgor a subventionnées. L'hypothèse selon laquelle le Zemgor aurait été, du moins dans la première moitié des années 1920, une sorte de relais administratif du Conseil des ambassadeurs se dégage de la consultation des archives (cf. par exemple les directives du Conseil concernant les modalités de prise en charge des armées du général Wrangel).

Nombre de rapports et de correspondances font état d'un partage de compétences entre les différentes organisations (au premier chef entre la Croix-Rouge et le Zemgor, mais aussi entre les différents comités caritatifs représentant les intérêts russes en exil) (re)constituées à l'étranger. Un souci d'efficacité, de rationalisation des tâches paraît dominer dans ce partage des compétences mais d'autres préoccupations (d'ordre politique, volonté de mobiliser différents réseaux, etc.) doivent être prises en considération dans l'organisation très hiérarchisée de la vie communautaire telle qu'elle se constitue au début des années 1920. Le Zemgor de Paris se place sous l'autorité du Conseil des ambassadeurs, contrôle ses représentants dans les pays européens, parraine un certain nombre d'associations (de secours à l'enfance notamment), exerçant ainsi ses prérogatives dans des contextes très diversifiés. Toutes les logiques de ce mode de fonctionnement par délégations associatives dans des secteurs très variés demandent encore à être explicitées et le dépouillement des archives de Leeds devrait contribuer à les éclairer. Des modalités spécifiques d'obtention de subventions, signalées dans diverses correspondances, peuvent, par exemple, expliquer la création d'associations d'entraide ad hoc à l'initiative du Zemgor. Le rôle pivot de ce dernier dans le vaste domaine de l'entraide et de la vie culturelle de l'émigration peut ainsi permettre de préciser les différentes stratégies et les différents mobiles qui ont présidé à l'organisation communautaire en exil.

\section{L'action éducative et culturelle du Zemgor}

Très marqué par le contexte de la guerre civile et des évacuations, le Zemgor a cherché à donner, par ses formes d'encadrement, une dimension humanitaire à ses actions éducatives et culturelles. Ce constat se vérifie tout particulièrement dans l'une des actions phares du Zemgor que fut l'aide à l'enfance. En mettant en place 
un important réseau d'institutions scolaires dans les principaux pays d'accueil des réfugiés, il s'est attaché à fournir aux enfants les moyens matériels de survie, de reconstruction psychologique, de formation dans la culture originelle, mais aussi d'adaptation au pays d'accueil. Dans cette perspective il a particulièrement favorisé l'organisation d'internats (souvent en milieu rural) qui, vers le milieu des années 20, avaient à leur charge près de 5000 enfants (sur les 20000 environ se trouvant en émigration).

Les correspondances des comités locaux avec le Comité directeur nous renseignent sur la genèse des institutions scolaires mais aussi sur l'évolution de leur fréquentation, sur le mode de gestion et de financement (subventions partielles ou totales des États d'accueil notamment), ainsi que sur le personnel éducatif et ses conditions de travail.

Jusque dans les années 1930, l'action éducative occupe une place prépondérante dans les dépenses annuelles des différents comités du Zemgor. Certains comités, en particulier ceux de Belgrade, de Sofia et de Prague, envoient des rapports circonstanciés sur leurs activités dans le domaine de l'éducation. Il apparaît ainsi que le Zemgor suit les programmes établis en Russie avant 1917 tout en témoignant d'un esprit d'ouverture à certaines méthodes scolaires des pays d'accueil. En Tchécoslovaquie par exemple, les écoles du Zemgor se sont inspirées des écoles tchécoslovaques dites « réformées » qui insistaient sur les matières scientifiques et les langues modernes. Les équipes enseignantes du Zemgor ont ainsi œuvré dans un souci de renouvellement pédagogique, se nourrissant des travaux et des débats de pédagogues russes et occidentaux, ainsi qu'en rendent compte les archives de Leeds, notamment à travers le Bulletin du Bureau pédagogique de Prague (collection complète), dont le Zemgor était membre fondateur. Parmi les différentes initiatives engagées par le Bureau pédagogique, on peut citer l'étude réalisée sur les conséquences psychologiques de la guerre civile et de l'exil chez les enfants, à partir d'enquêtes menées auprès des élèves des internats russes à l'étranger.

En dehors de son action éducative proprement dite, le Zemgor a étendu son action à la formation professionnelle et supérieure, sous des formes diverses : organisation de cours (enseignement de la langue du pays d'accueil, session de préparation à l'entrée dans les universités), mise en place de cycles de formation professionnelle (mécanique, agronomie, conduite), soutiens financiers aux études supérieures et aux intellectuels (aide à l'obtention de bourses d'État ou aides directes, sous la forme de prêts alloués à des particuliers ou des associations comme l'Union des écrivains et des journalistes russes en France).

La contribution du Zemgor au développement de la vie universitaire russe à l'étranger s'est concrétisée dans le soutien à la création de plusieurs institutions de l'entre-deux-guerres, telles les universités populaires russes de Paris et Prague, la faculté de droit de Prague, qui acquirent une forte renommée en raison de la qualité du corps professoral, constitué par des personnalités comme A. A. Kizevetter, P. B. Struve, P. I. Novgorodcev, G. Florovskij, D. D. Grimm, etc. De la sorte, le Zemgor a notamment stimulé le lien inter-générationnel entre les intellectuels et la jeunesse de l'émigration, qui était l'une des ses préoccupations majeures. 
Dans le domaine très vaste de la vie culturelle en émigration, le rôle du Zemgor s'est illustré à la fois par des actions ponctuelles (organisation de conférences, soutien à l'organisation d'expositions, aux journées de la culture russe, etc.) et des actions durables, comme la constitution de bibliothèques. Il contribua ainsi à la création d'un important réseau de bibliothèques, à l'origine conçues comme un instrument de soutien psychologique et moral, en particulier dans les camps d'internement des îles de Turquie où plusieurs milliers de civils et militaires avaient été cantonnés après les grandes évacuations de 1920. Par la suite, ces bibliothèques sont devenues un moyen important de diffusion de la production intellectuelle de l'exil (livres, revues, journaux).

Dans le domaine des actions durables, il faut faire une place à part à la création, à Prague, du Centre d'archives historiques russes à l'étranger (Russkij zagranničnyj istoričeskij arhiv). Ce centre de conservation avait pour vocation de rassembler tous les matériaux disponibles sur la révolution, la guerre civile et l'émigration. L'initiative trouva un large écho grâce à l'existence d'un réseau très dynamique de correspondants dans les différents pays de la diaspora et, de fait, la collection dite des «Archives de Prague» demeure l'une des sources majeures pour l'étude de l'émigration.

Les archives de Leeds devraient permettre d'évaluer ce champ d'activités éducatives et culturelles, de préciser le rôle des responsables du Zemgor dans l'organisation et le soutien des différents cercles de l'intelligentsia russe à l'étranger, notamment par la mise en place de structures et d'activités permettant d'éviter le risque d'un déclassement professionnel.

\section{L'action sociale du Zemgor}

Outre les domaines éducatif et culturel, le Zemgor a défini son champ d'action dans l'aide à l'insertion des réfugiés, non seulement à travers la formation professionnelle (cf. supra), mais surtout à travers la mise en place de bureaux pour l'emploi, la création d'ateliers artisanaux, l'organisation du placement des réfugiés, sous forme de recrutement collectif ou individuel, dans des grandes entreprises ou dans des régions agricoles, en favorisant autant que possible les regroupements géographiques de réfugiés, la création de «colonies russes » auprès de l'organisme employeur. Cette activité a perduré tout au long de l'entre-deux-guerres contrairement à d'autres actions d'entraide ponctuelles réalisées dans la première moitié des années 1920 (organisation de soupes populaires en Pologne, collectes et distributions de vêtements, hébergements dans différents pays de la diaspora).

L'activité de placement du Zemgor constitue l'un des domaines où s'est le mieux exprimé l'héritage des pratiques expérimentées au cours de la Première Guerre mondiale et poursuivies dès les premiers mois de l'exil à travers l'évacuation des réfugiés de Constantinople vers les pays environnants (Bulgarie, Yougoslavie principalement). Dans l'action d'aide au placement, le Zemgor s'est constitué comme intermédiaire entre les réfugiés en quête de travail et les entreprises, ou 
les organismes d'embauche. Cette activité a nécessité une importante collecte d'informations. Outre les différents rapports sur l'état des colonies russes (en particulier en Europe du Sud-Est), le Zemgor a été amené à établir des listes, des fichiers fournissant de nombreuses indications sur le parcours des demandeurs d'emploi (formation, expérience professionnelle en Russie, à l'étranger, etc.) et leur souhait d'insertion. Le chercheur dispose ainsi de sources quantitatives non négligeables pour étudier les parcours sociaux des réfugiés.

Une mention particulière doit être faite concernant l'activité du Zemgor dans le placement agricole, notamment dans le Sud-Ouest de la France. L'action de mise en valeur des terres par le placement d'agriculteurs étrangers dans cette région avait d'abord été entreprise à l'initiative du gouvernement français avant d'être confiée au BIT qui agit en collaboration directe avec le Zemgor. Ce dernier fournissait des candidatures pour la reprise d'exploitations en métayage tandis que le BIT s'engageait à accorder des prêts (dont les modalités de remboursements étaient administrées par le Zemgor) pour l'installation et l'acquisition d'outillage. De très nombreuses correspondances avec le BIT, le service de main-d'œuvre du ministère de l'Agriculture, les exploitants français, les agriculteurs russes permettent de reconstituer cette action et sa portée.

L'action sociale du Zemgor s'est également développée dans le domaine de l'assistance juridique sous la forme de bureaux de consultation, de permanences organisées dans diverses associations professionnelles russes afin de résoudre les difficultés, essentiellement administratives, rencontrées par les réfugiés. À titre individuel, plusieurs des responsables du Zemgor, tel Ja. Rubinštejn, jouèrent un rôle important dans le processus d'élaboration du statut du réfugié au sein de la commission consultative du Haut commissariat aux réfugiés, témoignant de la centralité du Zemgor, comme groupe d'influence dans la défense et l'institutionnalisation du réfugié sur la scène européenne.

\section{III — Le Zemgor et le développement de la politique humanitaire en Europe}

L'étude du Zemgor a surtout été envisagée jusqu'ici dans le contexte de l'histoire communautaire de l'émigration russe, mais elle mériterait également d'être resituée dans le développement de l'activité politique humanitaire moderne qui a caractérisé l'après-guerre. Dans quelle mesure, en effet, les stratégies, les orientations définies par le Zemgor tant à l'échelle des gouvernements, des organismes internationaux au sein de la Société des nations, qu'à l'échelle des autres organisations non gouvernementales, reflètent-elles cette émergence de l'acteur humanitaire dans le fonctionnement de l'État-providence ? De même, pour singulière que soit la trajectoire du Zemgor - d'administrations locales russes fédérées dans le contexte de la Grande Guerre à la création d'une organisation non gouvernementale diasporique - , ne porte-t-elle pas l'empreinte, plus générale dans les organisations humanitaires de l'époque, de l'expérience de la gestion sociale en temps de conflit, dans ses motivations, ses orientations au cours de l'après-guerre ? 


\section{Le rôle du Zemgor à la Société des nations}

Dans ces perspectives, le Zemgor peut, en premier lieu, être envisagé comme groupe de pression auprès des grands organismes européens, comme le HCR ou le BIT dont il fut à la fois un interlocuteur officiel pour certaines actions engagées et un interlocuteur indirect dans d'autres secteurs, tel le domaine juridique. Comment s'est exercée cette influence, quels ont été les réseaux constitués entre Genève et les responsables de l'organisation, sachant que de nombreuses relations avaient été tissées antérieurement, au cours de la Grande Guerre principalement, puis à titre de conseils, en particulier dans la question des prisonniers de guerre russes à rapatrier, à partir de 1918 ? Les nombreuses correspondances contenues dans les archives de Leeds devraient, à ce propos, fournir un éclairage complémentaire aux archives de la Société des nations à Genève et à la collection SDN conservée au GARF.

L'étude des relations, de la collaboration entre le Zemgor et ces organismes conduit à s'interroger sur le rôle des organisations humanitaires dans la définition, l'orientation de l'aide et la conception même du réfugié telles qu'elles se sont instituées dans la sphère européenne, avec la création, en 1921, du premier Haut commissariat, à l'origine exclusivement conçu pour l'assistance aux Russes en exil.

\section{LeZemgor et les interlocuteurs étatiques}

Outre les institutions européennes, le Zemgor s'est également trouvé en contact étroit avec certains gouvernements dont, au premier chef, le gouvernement tchécoslovaque et le gouvernement français, comme l'attestent nombre de correspondances, en particulier dans l'action de placement, mais aussi dans l'échange plus général d'informations et la formulation de diverses requêtes. Contrairement à une opinion fort répandue concernant l'attitude réfractaire de l'État français face aux regroupements des étrangers, ces échanges témoignent clairement de la contribution des pouvoirs publics à l'élaboration d'un lien communautaire parmi les exilés, soutenant les efforts du Zemgor dans la création de «colonies russes » en France, en particulier en province. De même que pour les organisations européennes, l'étude du lobbying des responsables du Zemgor à l'égard des acteurs étatiques suppose de reconstituer les relations entre Russes et Français (à travers par exemple le rôle d'hommes pivots tels que Albert Thomas, Marcel Paon, M. Hainglaise, etc.).

\section{Le Zemgor dans les réseaux associatifs d'assistance en Occident}

Enfin, pour resituer le Zemgor dans le contexte du développement de l'action humanitaire de l'entre-deux-guerres, il importe de prendre en considération la multiplicité de ses liens verticaux noués avec les différentes organisations, nationales et internationales, d'aide, en particulier dans le domaine de l'assistance à l'enfance, aux intellectuels, secteurs qui ont fait l'objet d'une attention particulière 
et ont été fortement soutenus par la SDN. De ce point de vue, les sources sont nombreuses. Il s'agit, en premier lieu, des rapports, des correspondances du Zemgor avec l'ensemble des organisations représentées au sein du Comité consultatif des organisations privées (CCOP) mis en place à la SDN au début des années 1920, dans lequel figurent, outre les organisations russes, des associations d'assistance juives, arméniennes, ainsi que de nombreux comités caritatifs nationaux ou internationaux. L'analyse de ces sources permet de reconstituer les formes de collaboration développées et d'apprécier leur efficacité.

En dehors du CCOP, le Zemgor s'est engagé dans différents partenariats, par exemple avec des associations d'aide aux étudiants juifs ; il a, de même, bénéficié de fonds spécifiques en provenance d'organismes caritatifs américains, comme en témoignent diverses correspondances. À partir des archives du Zemgor de Leeds, il semble ainsi possible de resituer le Zemgor dans un tissu relationnel extrêmement divers et étendu qui atteste de l'importance prise par les associations privées dans l'assistance sociale aux étrangers.

Le fonds du Comité directeur du Zemgor des archives de Leeds, en venant compléter les autres archives existantes, devrait permettre, à terme, de restituer au Zemgor la place et la visibilité qui étaient les siennes dans l'émigration. La richesse documentaire de ce fonds, pour les périodes de l'émigration, mais aussi de la guerre civile, devrait encourager les chercheurs à aborder l'histoire de cette organisation dans un souci de continuité avec les travaux existants ou en cours sur le rôle social et politique des anciennes administrations locales russes des zemstva et des villes et de leurs Unions. On peut ainsi espérer que l'étude du Zemgor, à travers ses pratiques administratives, ses réseaux, les parcours de ses responsables et ses formes d'engagement dans l'action sociale et culturelle, permettra de rendre compte à la fois de l'importance de l'expérience acquise en Russie avant la Révolution et des mutations imposées par les changements radicaux de contexte que furent la guerre civile et l'émigration.

Centre d'études du monde russe, soviétique et post-soviétique

54, boulevard Raspail

75006 Paris

gousseff@ehess.fr

pichon-bobrinskoy@wanadoo.fr 